\title{
Neoterminal ileal blood flow after ileocolonic resection for Crohn's disease
}

\author{
W J Angerson, M C Allison, J N Baxter, R I Russell
}

\begin{abstract}
Endoscopic laser Doppler flowmetry was used to measure neoterminal ileal blood flow in 16 patients who had undergone ileocolonic resection for Crohn's disease and had since remained clinically and biochemically free of disease, and eight control patients who had undergone similar surgery for colonic carcinoma. Four patients with clinically active Crohn's disease of the terminal ileum were also studied. Neoterminal ileal recurrence in those with inactive Crohn's disease was graded endoscopically. The median and minimum of five local blood flow measurements performed in each patient were inversely correlated with the endoscopic recurrence grade $(r=-0.52$, $\mathrm{p}=0.04$ and $\mathrm{r}=-0.63, \mathrm{p}=0.01$ respectively). Relative to the control group, median blood flow was non-significantly lower in the inactive Crohn's disease group as a whole $(p>0.05)$ but was significantly reduced in patients with active disease $(p=0.02)$. A progressive reduction in tissue perfusion may accompany recurrence of Crohn's disease while at a subclinical stage.

(Gut 1993; 34: 1531-1534)
\end{abstract}

Recent studies of resection specimens from patients with Crohn's disease have shown inflammatory microvascular occlusion, apparently secondary to mesenteric vasculitis. ${ }^{1}$ The same authors have localised most granulomas in Crohn's disease to the wall of intestinal blood vessels. $^{2}$ The identification of focal microvascular injury within and adjacent to grossly normal mucosa led to their proposal that vascular compromise is central to the pathogenesis of Crohn's disease. It remains possible, however, that these abnormalities are simply a consequence of small vessels becoming involved in the chronic inflammatory process.

Several colonoscopic studies have drawn attention to the development of subclinical lesions within the neoterminal ileum after ileocolonic resection for Crohn's disease. ${ }^{3-7}$ Aphthoid ulcers and neoterminal ileitis develop in the early postoperative months in about $70 \%$ of patients. Although it was initially considered that these endoscopic signs were features of disease recrudescence, ${ }^{4}$ careful perioperative and postoperative surveillance studies have established that the lesions represent true disease recurrence. ${ }^{67}$ If microvascular occlusion plays a primary role in the pathogenesis of the disease, reduced blood flow would be expected in association with these subclinical manifestations of recurrence. This would be in contrast to the increased blood flow normally associated with an inflammatory response, as has been observed in ulcerative proctitis. ${ }^{8}$

Endoscopic laser Doppler flowmetry was used to perform a controlled study of neoterminal ileal blood flow in patients whose Crohn's disease had remained clinically and biochemically inactive after ileocolonic resection. The control group consisted of patients who had undergone similar surgery for carcinoma of the right colon. For secondary comparison, a small group of patients with clinical, biochemical, and endoscopic evidence of active Crohn's ileitis was also investigated.

\section{Patients and methods}

\section{CROHN'S DISEASE PATIENTS}

Seventeen asymptomatic patients who had previously undergone ileocolonic resection for Crohn's disease agreed to participate in this study. One was subsequently excluded because it proved impossible to advance the colonoscope as far as the anastomosis in this patient. There were five men and 11 women with a median age of 40 years (range 21-64 years). The diagnosis was based on typical clinical and histological features. The original operation had been performed a median of four years (range 1-14 years) before this study. Three patients had developed anastomotic disease recurrence and had required a further resection and reanastomosis 8-14 years before this study. Patients were not included if they had required corticosteroid treatment for active disease after their most recent resection. None had clinical or biochemical evidence of disease activity at the time of study (Best activity index $<150^{\circ}$ and serum $\mathrm{C}$ reactive protein $<10 \mathrm{mg} / \mathrm{l})$.

Four patients with clinically active Crohn's ileitis were also studied. They comprised one man and three women with median age of 25 years (range 18-33 years) and a median Best activity index of 250 (range 220-340). One of these patients had undergone ileocolonic resection for Crohn's disease five years before this study.

\section{CONTROL PATIENTS}

Eight patients with a history of ileocolonic resection for carcinoma of the right colon agreed to undergo blood flow studies during their planned surveillance colonoscopy. There were five men and three women with a median age of 67 years (range 51-79 years). The resection had been performed a median of three years previously (range 3-8 years). 
METHODS

After standard orthograde bowel preparation, each patient received intravenous diazepam and pethidine, the doses being titrated according to the response. The colonoscope was advanced to the ileocolonic anastomosis and the endoscopist graded the appearance of the neoterminal ileum in each Crohn's disease patient according to the criteria of Rutgeerts ${ }^{6}$ as follows:

Grade 0 - no lesions;

Grade 1 - less than five aphthous lesions;

Grade 2 - five or more aphthous lesions separated by mucosa of normal appearance;

Grade 3 - diffuse aphthous ileitis with diffusely inflamed mucosa;

Grade 4 -diffuse inflammation with larger ulcers and anastomotic narrowing.

A single endoscopist (MCA) performed all grading.

Blood flow measurements were performed using a Periflux PF3 laser Doppler flowmeter (Perimed, Stockholm) with a $12 \mathrm{kHz}$ bandwidth and 3 seconds output time constant. The endoscopic flowmeter probe (model PF309) had optical fibres of $125 \mu$ diameter and $250 \mu$ centre-to-centre spacing. Constant gain was ensured by regular calibration using a colloidal latex suspension according to the manufacturer's recommendations.

Hyoscine- $\mathrm{N}$-butylbromide $(40 \mathrm{mg}$ ) was given intravenously immediately before measurements to minimise peristalsis. The flowmeter probe was passed through the biopsy channel of the endoscope and the tip was placed in gentle contact with the mucosa. Measurements were obtained at five locations around the circumference' of the ileum just proximal to the anastomosis in the inactive Crohn's disease and control groups, and in three to five inflamed areas of the terminal ileum or ileocaecal junction in patients with active disease. Measurements on ulcers and their immediate margins were avoided. The flowmeter output, in arbitrary perfusion units, was recorded over a period of 30-60 seconds at each location, and the mean of each such recording, ignoring easily identified artefacts due to peristalsis, was taken as a single measurement. Interpretation of blood flow recordings and endoscopic grading were performed in a mutually blind fashion.

The reproducibility of blood flow measurements performed in this way was assessed in separate patients by performing successive recordings at a single location. The mean coefficient of variation of 12 of these series of repeated measurements was $18 \%$.

The coefficient of variation of the measurements for each patient in the present study was calculated as a measure of within patient variability. The median and minimum of the readings for each patient were used for intergroup

Blood flow in arbitrary perfusion units expressed as median and interquartile range for the three groups of patients

\begin{tabular}{lccl}
\hline & Controls & Inactive CD & Active CD \\
\hline Intrapatient median & $133(118-180)$ & $123(95-155)$ & $67(53-97)^{\star}$ \\
Intrapatient minimum & $83(58-130)$ & $65(40-88)$ & $39(33-46)$ \\
\hline${ }^{\star} \mathrm{p}<0.05$ relative to controls (Mann-Whitney U test). \\
$\mathrm{CD}=$ Crohn's disease.
\end{tabular}

comparisons and for assessment of the relationship between blood flow and endoscopic grade. Statistical analysis was performed using the Mann-Whitney test and Spearman's rank correlation test as appropriate. Departures from the null hypothesis were assessed at the $5 \%$ significance level.

\section{Results}

The within patient coefficient of variation for blood flow measurements ranged from 12.5 to $63.0 \%$, and averaged $31 \cdot 9 \%$. It did not differ significantly between groups.

Blood flow measurements in the three groups are compared in the Table and in Figure 1. Median and minimum neoterminal ileal blood flow values in patients with inactive Crohn's disease were lower than in non-Crohn's disease controls but the differences were not statistically significant $(p>0.05)$. In patients with clinically active Crohn's disease, blood flow was lower than in both of the other groups, although only the difference in median values relative to the control group was significant $(p=0 \cdot 02)$

There was an inverse correlation between the endoscopic grade of recurrence in patients with inactive Crohn's disease and both median and minimum blood flow values $(r=-0.52, p=0.04$, and $r=-0.63, p=0.01$ respectively: Fig 2 ). In the subgroup of patients with grade 2 recurrence or higher, the contrast with controls approached statistical significance $(p=0.05$ and $p=0.07$ for median and minimum values respectively). Blood flow was not significantly correlated with age or length of time since surgery in any of the groups.
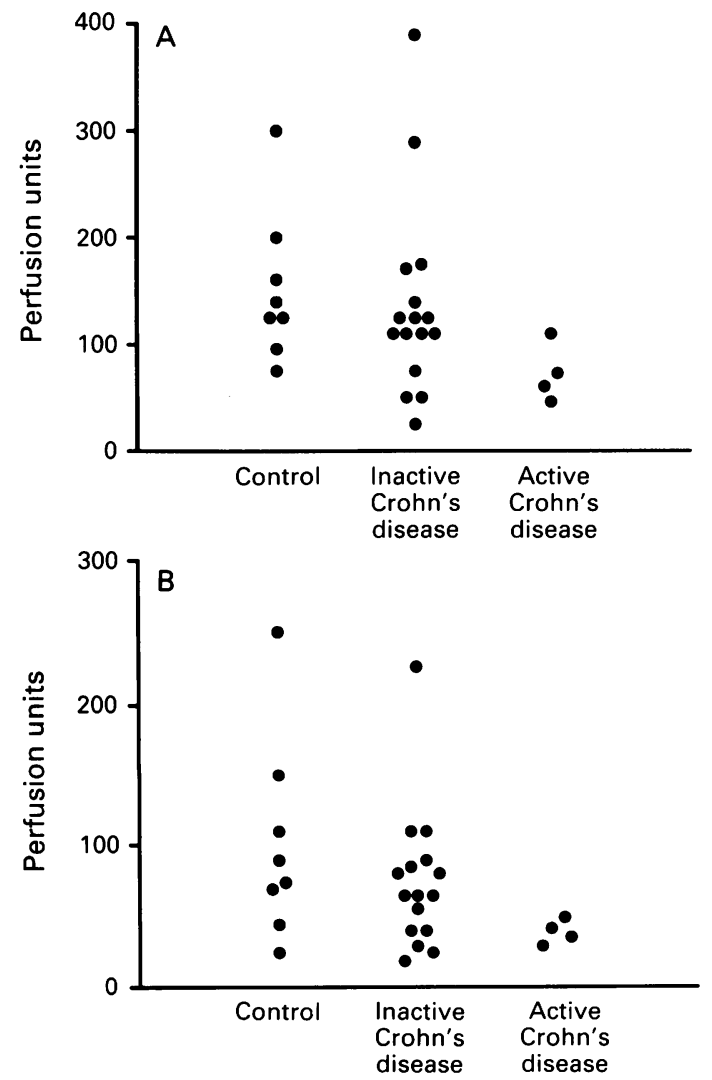

Figure 1: (A) Intrapatient median and $(B)$ intrapatient minimum blood flow values in the three groups of patients. 


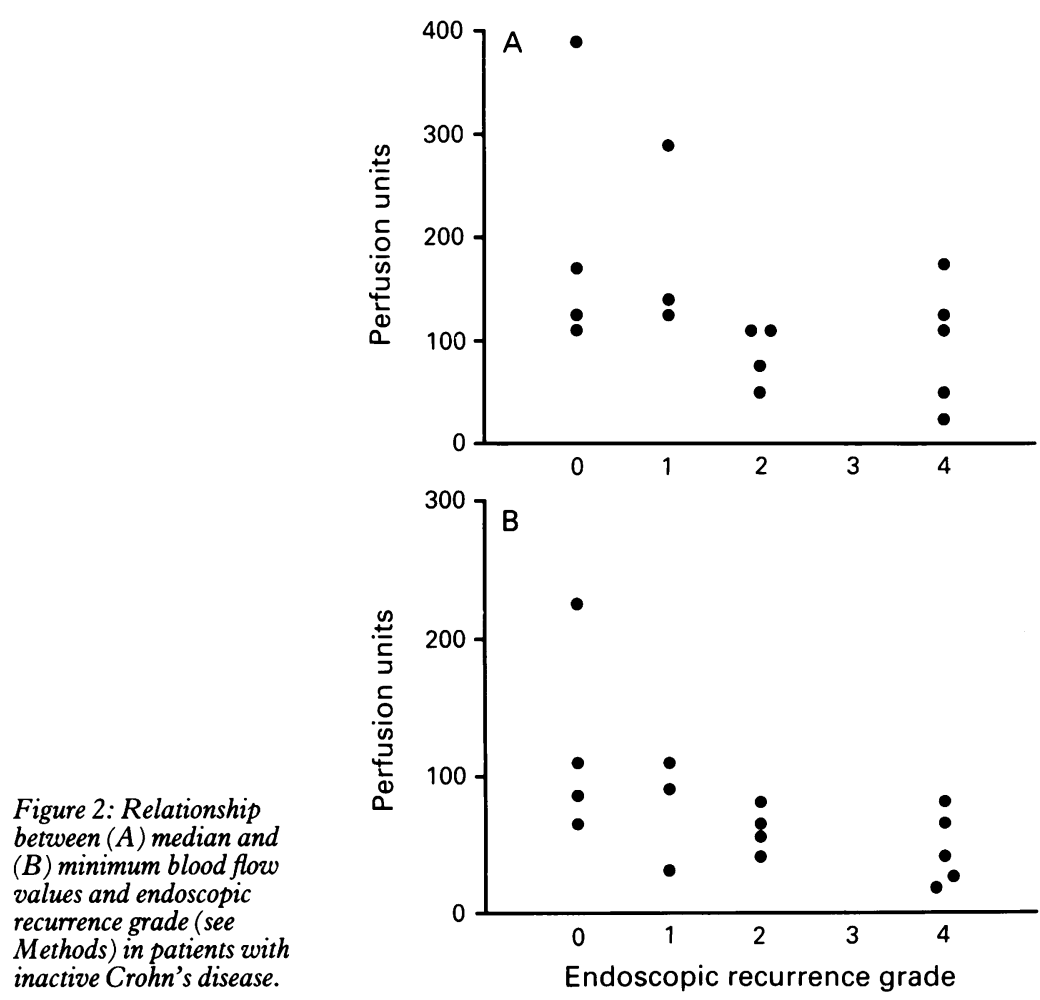

Discussion

This study has shown that after ileocolonic resection for Crohn's disease, neoterminal ileal blood flow as measured by laser Doppler flowmetry falls as the endoscopic recurrence severity grading increases. These changes precede clinical and biochemical evidence of disease recurrence. Patients with higher grades of endoscopic recurrence (at least five aphthous ulcers) and those with active Crohn's ileitis had similar levels of blood flow, which were lower than in controls.

There have been few previous studies of intestinal blood flow in Crohn's disease. Su et al ${ }^{10}$ using endoscopic reflectance spectrophotometry, suggested that blood flow was increased in affected regions of the colon in patients with active Crohn's disease. Hulten et $a l^{11}$ reported that colonic blood flow as measured by intraoperative ${ }^{85} \mathrm{Kr}$ clearance was increased in severe diffuse Crohn's colitis but reduced in chronic segmental disease. In Crohn's ileitis, the same authors found flow to be normal in early stage and reduced in late stage disease, and in both the colon and ileum reduced blood flow was associated with submucosal fibrosis and decreased vascularity. Quantitative fluorescent radiography has confirmed the reduction in the microvascular volume in segmental Crohn's disease. ${ }^{12}$ These observations supported long standing speculation that vascular insufficiency could be a pathogenic factor, but suggested that vascular and haemodynamic changes were variable and dependent on the stage and pattern of disease.

More recent histopathological and microvascular studies suggest that vascular involvement may occur at an earlier stage and play a more important role in the disease process than had previously been supposed. Wakefield $e t$ al have shown a multifocal occlusive vasculitis in resected bowel affected by Crohn's disease,' and have shown that a high proportion of granulomas within the bowel wall directly involve damaged blood vessels. ${ }^{2}$ As vascular lesions were observed in macroscopically normal areas of bowel, and adjacent to and in continuity with normal blood vessels, it was proposed that the vasculitis was a primary process and that the macroscopic and histological features of Crohn's disease could be explained by multifocal intestinal infarction.

Although the present study suggests that a reduction in blood flow may precede disease recurrence as defined by clinical and biochemical criteria, it does not support the suggestion that a perfusion defect is an essential precursor to mucosal ulceration. We analysed minimum blood flow values because of their potential to show the presence of focally ischaemic areas, but found a large overlap between Crohn's disease and control patients. Since the laser Doppler recordings represent highly localised measurements, it remains possible that the most poorly perfused areas escaped detection in some patients. Another possibility is that ischaemia progresses to ulceration so rapidly that it is unlikely to be observed in isolation.

We were unable to show a significant difference in blood flow between the control group and the inactive Crohn's disease group as a whole. It is possible that a greater difference would have emerged if we had been able to age-match the Crohn's disease and control groups, as age has been shown to be inversely correlated with intestinal blood volume. ${ }^{12}$ Another factor masking differences between groups was the wide variation in median blood flow values between individual patients. This was comparable with the interpatient variation reported by others using laser Doppler, venous outflow collection, and isotope clearance techniques in the gastrointestinal tract. 113

Variations in laser Doppler readings between different measurement sites within a given patient may be due to heterogeneity of flow or to factors degrading the reproducibility of measurements. The inter-site coefficient of variation in this study (32\%) was substantially higher than was observed when measurements were repeated at the same site (18\%), suggesting that much of the apparent heterogeneity was genuine. Inter-site variation in laser Doppler flow measurements has been shown to be partly related to the small dimensions of the measurement volume, ${ }^{1+}$ which make the results highly sensitive to local non-uniformity of microvascular perfusion.

An important question in the interpretation of the laser Doppler readings is the depth of the measurement region. Intraoperative studies by Ahn et $a l^{1315}$ showed a good correlation between laser Doppler flowmetry recordings and measurements of total venous outflow in the feline and human small bowel, and provided direct evidence that the measurements reflected blood flow throughout the full thickness of the bowel wall in both species. Those studies utilised a probe with $700 \mu$ diameter optical fibres, however, whereas the probe in the present study had narrower, more closely spaced fibres, and this would be expected to reduce the effective 
measurement depth. ${ }^{16}$ Johansson et al found that interposition of unperfused tissue of $2.4 \mathrm{~mm}$ thickness between the bowel and a probe with similar geometry to our own completely abolished the blood flow signal. ${ }^{16}$ This suggests that the depth of measurement in our study did not exceed $2.4 \mathrm{~mm}$, and that the measurements thus primarily represent mucosal perfusion, with a possible submucosal contribution attenuated by depth. Thickening of all layers of the bowel wall is a feature of Crohn's disease, a factor perhaps further weighting the recordings towards mucosal as opposed to submucosal and muscularis blood flow. Since the mucosa is normally the most richly perfused tissue layer, this factor is unlikely to have artificially promoted either the observed reduction in flow in patients with active disease, or the negative correlation between flow and disease severity in asymptomatic patients. However, it could have hidden a greater reduction in flow in the deeper layers of the bowel wall, which may be the main site of vascular compromise..$^{12} 12$

This study suggests that blood flow disorders are present in recurrent Crohn's disease that is subclinical, and are related to its endoscopic grade of severity. This is consistent with anatomical evidence of microvascular occlusion and with the hypothesis that reduced tissue perfusion is a contributory factor in the pathogenesis of Crohn's disease, although proof of a cause/effect relationship remains a difficult task. Endoscopic laser Doppler flowmetry provides a suitable means of investigating haemodynamic aspects of the disease process, and further advances may be made by performing longitudinal studies.
1 Wakefield AJ, Sawyerr AM, Dhillon AP, Pittilo RM, Rowles PM, Lewis AAM, et al. Pathogenesis of Crohn's disease: multifocal gastrointestinal infarction. Lancet 1989; ii: 105762

2 Wakefield AJ, Sankey EA, Dhillon AP, Sawyerr AM, More L, Sim $R$, et al. Granulomatous vasculitis in Crohn's disease. Gastroenterology 1991; 100: 1279-87.

3 Rutgeerts P, Geboes K, Vantrappen G, Kerremans R, Coenegrachts JL, Coremans G. Natural history of recurrent Crohn's disease at the ileocolonic anastomosis after curative surgery. Gut 1984; 25: 665-72.

4 Tytgat GNJ, Mulder CJJ, Brummelkamp WH. Endoscopic lesions in Crohn's disease early after ileocaecal resection. Endoscopy 1988; 20: 260-2.

5 Lescut D, Vanco D, Colombel JF, Bonniere P, Quandalle P, Lecompte-Boucke $M$, et al. Influence des lésions endoscopiques d'amont sur les récidives endoscopiques anastomotiques (rea) au cours de la maladie de Crohn (MC). Gastroenterol Clin Biol 1990; 14: A20.

6 Rutgeerts P, Geboes K, Vantrappen G, Beyls J, Kerremans R, Hiele $M$. Predictability of the postoperative course of Crohn's disease. Gastroenterology 1990; 99: 956-63.

7 Olaison G, Smedh K, Sjodhal R. Natural course of Crohn's disease after ileocolic resection: endoscopically visualised ileal ulcers preceding symptoms. Gut 1992; 33: 331-5.

8 Srivastava ED, Russel MAH, Feyerabend C, Rhodes J. Effect of ulcerative colitis and smoking on rectal blood flow. Gut 1990; 31: 1021-4.

9 Best WR, Becktel JM, Singletom JW, Kern F, Summers RW, Switz DM, et al. Development of a Crohn's disease activity Switz DM, et al. Development of a Croh
index. Gastroenterology 1976; 70: 439-44.

$10 \mathrm{Su}$ KC, Leung FW, Guth PH. Assessment of mucosal haemodynamics in normal human colon and patients with inflammatory bowel disease. Gastrointest Endosc 1989; 35: 22-7.

11 Hulten $\mathrm{L}$, Lindhagen $\mathrm{J}$, Lundgren $\mathrm{O}$, Fasth $\mathrm{S}$, Ahren $\mathrm{C}$. Regional intestinal blood flow in ulcerative colitis and Crohn's disease. Gastroenterology 1977; 72: 388-96.

12 Carr ND, Pullan BR, Schofield PF. Microvascular studies in non-specific inflammatory bowel disease. Gut 1986; 27: 542-9.

13 Ahn $\mathrm{H}$, Lindhagen J, Nilsson GE, Oberg PA, Lundgren $\mathrm{O}$ Assessment of blood flow in the small intestine with laser
Doppler flowmetry. Scand $\mathcal{F}$ Gastroenterol 1986; $21: 863-70$.

14 Salerud EG, Nilsson GE. Integrating probe for tissue laser Doppler flowmeters. Med Biol Eng Comput 1986; 24:415-9.

15 Ahn H, Lindhagen J, Nilsson GE, Salerud EG, Jodal M, Lundgren $O$. Evaluation of laser Doppler flowmetry in the assessment of intestinal blood flow in cat. Gastroenterology 1985; 88: 951-7.

16 Johansson $\mathrm{K}$, Jakobsson $\mathrm{A}$, Lindahl $\mathrm{K}$, Lindhagen $\mathrm{J}$, Lundgren $\mathrm{O}$, Nilsson GE. Influence of fibre diameter and probe geometry on the measuring depth of laser Doppler Clin Exp 1991; 10: 219-29. 